Politicas socio-educativas y terRitorio. Una descripción PReliminar. Páginas 149-162 en Revista de la Escuela de

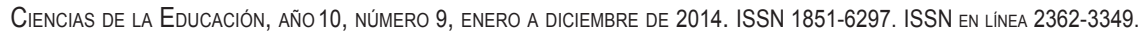

\title{
POLÍTICAS SOCIO-EDUCATIVAS Y TERRITORIO. UNA DESCRIPCIÓN PRELIMINAR
}

\author{
Por Mariana Beatriz Nemcovsky (Universidad Nacional de Rosario)*, Argentina. \\ mbnem@hotmail.com
}

Recibido: 28/06/2014 Aceptado: 27/08/2014

\section{Resumen}

En esta presentación (1) me interesa describir aspectos de las políticas socioeducativas diseñadas desde el Ministerio de Educación de la Provincia de Santa Fe atendiendo a los nexos que, para su aplicación, se plantean entre distintos planes tendientes a alcanzar la denominada "inclusión educativa" de niños y jóvenes, así como a las articulaciones territoriales previstas entre diferentes áreas del gobierno provincial y municipal de la ciudad de Rosario.

En primer lugar describo brevemente las políticas educativas formuladas e implementadas por el gobierno de la provincia de Santa Fe en los últimos años, para centrarme luego en algunas concepciones respecto de la escuela y la inclusión educativa que se desprenden de esas formulaciones. Particularmente me interesa analizar el modo en que se entraman esas concepciones con una perspectiva de territorialización en el diseño e implementación de las políticas.

Si bien trabajaré a escala de la provincia, entiendo tal como, he señalado en otros trabajos, que estas políticas, en nuestro presente histórico, en general, se expresan en consonancia con políticas socio-educativas formuladas en otros niveles contextuales: internacional, regional, nacional.

\section{Palabras Clave}

Políticas educativas - Escuela - Territorio - Inclusión - Desigualdad social.

* Doctora en Humanidades y Artes con mención en Antropología. Escuela de Posgrado FHyA. UNR. Licenciada en Antropología (orientación socio-cultural) Escuela de Antropología. FHyA UNR. 
Revista de la Escuela de Ciencias de la Educación, año 10, número 9, enero a diciembre de 2014. PÁginas 149-162. ISSN 1851-6297. ISSN EN LINEA 2362-3349. PoliticAS SOCIO-EDUCATIVAS Y TERRITORIO. UNA DESCRIPCIÓN PRELIMINAR. Mariana Beatriz Nemcovsky.

\begin{abstract}
In this presentation I am interested in describing aspects of the socio-educational politics worked out by the Ministry of Education of The Province of Santa Fe. That is why this description will be focused on the links which are shown between different programs so as to reach the so called "educational integration" for children and young people. In the same way, I am keen on analyzing the territorial ties which are foretold between the different areas of The Provincial Government and The Municipal Government of Rosario.

In first place I give a brief description of the educational politics which are formulated and carried into effect by the Government of The Province of Santa Fe in the last years. I will then bring into focus some ideas regarding the school and the educational integration which come from those formulations. Specifically, I'm interested in analyzing the way in which those ideas are entwined with a territorial perspective in the design and implementation of the politics.

Even though I will work on scale of the Province, I understand that those politics, in our historical present, are expressed in relation to socio-educational politics which are formulated in other contextual levels: international, regional, national.
\end{abstract}

\title{
Key Words
}

Educational politics - School - Territory - Integration - Social inequality.

\section{Políticas destinadas a "barrios problemáticos/prioritarios"}

En los últimos años se han diseñado un conjunto de políticas con el propósito de incidir en espacios socio-urbanos de la ciudad de Rosario y de la provincia, identificados como zonas o "barrios problemáticos".

De modo rápido y a los efectos sólo de mencionarlos, me interesa enunciar algunos procesos que se han combinado en nuestra ciudad y que, entiendo no pueden soslayarse a la hora de analizar las políticas socio-educativas en contexto. En primer lugar señalar que a esos espacios, hoy "priorizados"-en los que la desigualdad social, $y$, un conjunto de procesos relacionados, permea los cotidianos sociales- se incorporan visiblemente en los últimos años, circuitos delictivos vinculados a distintos negocios, entre ellos, al narcotráfico, y, que tales circuitos no se han gestado rápidamente. Tampoco la discriminación/estigmatización y racialización construidas socialmente respecto de los conjuntos sociales que los habitan y el sufrimiento social derivado de ello. Determinadas situaciones, que eran reconocidas/denunciadas por los pobladores de muchos barrios de la ciudad ya a mediados de los años 90' (2), como vinculadas a lo que hoy conceptualizamos como narcomenudeo para describirlas, daban cuenta de la presencia a escala de la ciudad de dichos circuitos narco-delictivos; sostenidos tal vez por un consumo ya significativo de algunas sustancias (3). A la vez, como sabemos, Rosario fue una de las ciudades con los más altos índices de desocupación de nuestro país, $18,3 \%$ de la población económicamente activaPEA-(4), al finalizar los noventa y hasta iniciarse el período que algunos llaman 
Revista de la Escuela de Ciencias de la Educación, año 10, número 9, enero a diciembre de 2014. Páginas 149-162. ISSN 1851-6297. ISSN en LINEA 2362-3349. PolíticAS SOCIO-EDUCATIVAS Y TERRITORIO. UNA DESCRIPCIÓN PRELIMINAR. Mariana Beatriz Nemcovsky.

de post-convertibilidad, como consecuencia de los procesos de transformación estructural que se profundizaron en esos años. Distintas generaciones de conjuntos sociales que fueron subalternizados y vulnerabilizados, expulsados del trabajo, de las organizaciones que nuclean/referencian a los trabajadores, han sufrido diferentes desplazamientos en el espacio urbano y habitan, en nuestro presente histórico, zonas de pobreza estructural en la mayoría de los distritos de la ciudad. Si bien aquellos índices relativos a la desocupación han disminuido significativamente, aún es importante el empleo "en negro", y, para algunos de esos sectores de la población, ha persistido la dificultad del acceso laboral y/o ha continuado la sub-ocupación o el empleo intermitente; lo que hace necesaria la intervención del estado nacional a través de políticas que resultan compensatorias, como los denominados planes sociales. Estas transformaciones fueron acompasadas por un clima de época que se hace fuerte desde los años 90, una "filosofía integral" (Borón, 2010), una concepción de la sociedad, tal es de lo que se trata el neoliberalismo, que permea el conjunto de las prácticas sociales. Una corriente ideológica caracterizada por elementos tales como el individualismo, la competitividad, la valoración suprema de lo efímero, las "fugas" y la "fluidez de lo líquido". "Valores y objetivos de una sociedad capitalista, consumista, individualista, dura y fuertemente competitiva" que "son similares a los valores y objetivos de los jóvenes" (Menéndez, 2010) que viven en contextos de desigualdad social y participan de circuitos de narcomenudeo. Aunque tal participación forme parte de lo que Eduardo Menéndez (2010) caracteriza como "estrategias de aguante", vividas por niños y jóvenes en términos de experiencias "laborales".

Estos procesos que han ido calando en una ciudad, que además es puerto, a la que desde la tecnologización de la producción de la soja, confluyen capitales, que según especialistas, como Adriana Rossi (2014), ingresan al negocio inmobiliario con escasos controles, favoreciendo el "lavado" de dinero de proveniencia dudosa, configuran el escenario en el que las políticas socio-educativas se implementan "en el juego no predecible con los sentidos locales" (Montesinos \& Sinisi, 2009). Escenario conceptualizado, en términos de "territorio" como espacio de confluencia y articulación de las políticas, que involucra los sentidos y prácticas construidos en el nivel de la vida cotidiana por los conjuntos sociales que lo habitan.

Las acciones diseñadas en las políticas como estrategias para operar en ese nivel suponen la concentración articulada de formulaciones de las órbitas provincial y municipal- retomando, más o menos explícitamente algunas políticas socioeducativas nacionales.

\section{Los programas socio-educativos a escala provincial}

Las políticas socio-educativas formuladas a escala de la provincia de Santa Fe se presentan en un "proyecto pedagógico provincial". Para llevarlo adelante 
Revista de la Escuela de Ciencias de la Educación, año 10, número 9, enero a diciembre de 2014. PÁginas 149-162. ISSN 1851-6297. ISSN EN LINEA 2362-3349. PolitICAS SOCIO-EDUCATIVAS Y TERRITORIO. UNA DESCRIPCIÓN PRELIMINAR. Mariana Beatriz Nemcovsky.

el Ministerio de Educación provincial ha formulado un conjunto de programas; algunos planteados como innovación pedagógica y otros como de formación continua (5). Estos programas, están destinados a la educación común y sus distintas modalidades (6), aunque se implementan de acuerdo con condiciones presupuestarias e infraestructurales, privilegiando los denominados "barrios prioritarios". En general están diseñados para ser implementados en determinados niveles educativos y sólo algunos están dirigidos a todos ellos. A la vez, aunque estén destinados a determinado nivel educativo, y se prioricen determinados barrios, se implementan segmentadamente en relación a las escuelas y al interior de ellas. Ello contribuye a generar interpretaciones de las docentes en términos de "lo desiguales que están las escuelas" (7).

Distintos programas socioeducativos son propuestos más o menos explícitamente como acciones planificadas en territorio para alcanzar la inclusión educativa; algunos están dirigidos a los sujetos de la experiencia escolar, alumnos de distintos niveles educativos así como a directivos y docentes. Otros incorporan al espacio escolar a los grupos familiares y/o sus acciones socioeducativas se fundan en la articulación con instituciones de la órbita municipal.

Entre los primeros podemos mencionar los programas vinculados con la transmisión/apropiación de elementos artísticos del campo de la música y la danza. Están aquellos que consisten en la conformación de Orquestas y Coros, dirigidos a niños y jóvenes, que se proponen "como modo de incluir y retener a los niños y jóvenes en las escuelas" y forman parte de un programa nacional (8). También a nivel de la Formación docente de música, se proponen Ensambles de percusión, canto y el movimiento expresivo. Este programa, si bien no contiene explícita en su formulación la noción de inclusión, apela fuertemente al trabajo territorial, entendiendo el "fenómeno musical en toda su complejidad, reconociendo en él, el valor representacional social, cultural y político... y anclando este trabajo en territorio con "el acompañamiento a cargo de equipos de referentes territoriales del programa consistente en visitas a escuelas/ aulas y encuentros zonales de apoyo para el trabajo de música en el aula" (9).

En el mismo sentido, la Feria de ciencia es caracterizada como "sustenta[da] en prácticas educativas y experiencias escolares inclusivas entendidas como la construcción colectiva de saberes socialmente relevantes" (10). Por su parte, el programa Escuela Abierta constituye para el Ministerio una instancia de formación docente permanente para el que la calidad educativa "está indisolublemente ligada a la inclusión socioeducativa de todos los santafesinos" (11).

Entre aquellos programas cuyos objetivos suponen el trabajo aunado entre equipos del Ministerio de Educación y de la Municipalidad en el territorio, "en escuelas y en los barrios", Sabores y saberes constituye una "propuesta que implica la generación de espacios de comensalidad en la escuela y los hogares" (12). Se implementa en las escuelas que tiene comedor escolar, a las que concurren niños y jóvenes que en gran parte asisten a él, "integrando 
Revista de la Escuela de Ciencias de la Educación, año 10, número 9, enero a diciembre de 2014. PÁginas 149-162. ISSN 1851-6297. ISSN en LINEA 2362-3349. PolíticAS SOCIO-EDUCATIVAS Y TERRITORIO. UNA DESCRIPCIÓN PRELIMINAR. Mariana Beatriz Nemcovsky.

el espacio educativo y el comedor, porque se trata de "mirarse a la cara cuando se consume el alimento, de compartir, convivir". Plantea su trabajo con el personal directivo, docente, no docente y la comunidad, y a partir del 2013, contempla la incorporación de equipos de los Centros de Convivencia Barrial y del Ministerio de Educación ya que uno de sus ejes es la territorialidad. Constituye "una propuesta que se renueva.., cumpliendo con la meta de Educación que es alcanzar la calidad educativa, pero cuando hablamos de calidad esto implica inclusión y convivencia en cada una de las escuelas" (13). Otra de las formulaciones, que reúnen en sus diseños la articulación territorial entre equipos de distintas instituciones del estado y la preocupación por la convivencia, la integración, el compartir a nivel del espacio escolar, es la denominada El Patio. Se entiende como "un espacio socio-educativo, una propuesta para que el barrio se encuentre, permitiendo así a los niños, jóvenes y adultos vivenciar, protagonizar y participar.... para recuperar vínculos, autoestima y conocimientos que reconstruyan el sentido de lo colectivo. Es un encuentro cotidiano para aprender a hacer, a ser, a convivir". Sus objetivos se plantean en relación a "Igualar las condiciones de aprendizaje, fortalecer la retención escolar" y "ocupar el tiempo libre" (14).

Algunos programas como Aprendizajes en juego, entendido como generador de "un movimiento que vuelva a enlazar escuela y sociedad en un vínculo de esperanza y emoción que nunca sucumbió del todo... como una instancia de participación e integración"; Ronda de palabras cuyo objetivo es "promover el ejercicio del pensamiento como práctica reflexiva y la comunicación de las ideas en un ámbito de libertad y confianza"; De ESI sí se habla, relativo a la Educación Sexual Integral de niños/niñas y adolescentes, entre otros, si bien no contienen explícitamente en sus formulaciones apelaciones a la inclusión educativa y/o al trabajo territorial, se implementan a partir de las caracterizaciones institucionales producidas por equipos que participan del trabajo territorial. Dicha implementación muy requerida a veces por las docentes, se produce de acuerdo a las decisiones tomadas en relación con esas instancias territoriales, de modo segmentado, al interior, y entre las escuelas de los barrios favoreciendo experiencias diferenciales.

"Ronda de Palabras ayuda mucho con 2do grado, en otros grados no pudimos, quisimos, les dijimos a las chicas del socio-educativo mandamos nota, pero no, están desiguales las escuelas" (15).

El conjunto de las acciones descriptas la enseñanza de la música, la feria de ciencias, la formación docente, a través de Escuela Abierta, Sabores, y Saberes, El Patio, tienen, como señalamos, un fuerte énfasis en la inclusión y retención educativa de la población de los barrios de la ciudad e involucran trabajo territorial para ello. A la vez, los programas que incorporan "la participación de la comunidad", y están destinados a los barrios en los que las escuelas 
Revista de la Escuela de Ciencias de la Educación, año 10, número 9, enero a diciembre de 2014. PÁginas 149-162. ISSN 1851-6297. ISSN EN LINEA 2362-3349. PoliticAS SOCIO-EDUCATIVAS Y TERRITORIO. UNA DESCRIPCIÓN PRELIMINAR. Mariana Beatriz Nemcovsky.

tienen comedor contienen una importante carga prescriptiva. Parecen partir de atributos negativos, de caracterizar la ausencia de algunas prácticas en los sujetos a los que se dirigen al proponer que se trata de "mirarse a la cara para consumir el alimento", que necesitan "prácticas higiénicas al producir el alimento", "encontrase, recuperar vínculos... aprender a hacer, a ser, a convivir."

Una de las formulaciones oficiales más emblemáticas en relación con la inclusión educativa es el Plan Vuelvo a Estudiar, dirigido a jóvenes que no han completado el nivel secundario, en el que se sostiene que "se hace necesario instalar la vulnerabilidad como criterio para dar prioridad a la inclusión educativa"(16). Se trata de un Plan que está diseñado para implementarse en dos fases, una al interior de la escuela y otra a través de la articulación territorial con instituciones del estado y de la sociedad civil.

Algunos programas de Innovación Pedagógica, tales como Escuela Abierta, así como otras acciones enmarcadas en lo que se conceptualiza desde el Ministerio como Líneas pedagógicas, expresan algunas concepciones que nos parece necesario analizar.

El "proyecto pedagógico" del Ministerio de Educación provincial concibe a la escuela como "un espacio público por ser un territorio de todos, de intercambio, de juego y convivencia y escenario de la cultura democrática" y caracteriza al sistema educativo como "antiguo y estructuralmente rígido." Por ello, se plantea la premisa "de dinamizar la estructura, dotarla de flexibilidad e introducir innovaciones educativas basadas en las experiencias vivenciales y en las competencias emocionales de los actores involucrados en los procesos de enseñanza-aprendizaje."

En primer lugar, la idea de una escuela como un espacio "de intercambio, de juego y convivencia y escenario de la cultura democrática" que "está siendo interpelada por problemáticas sociales que la atraviesan e impactan en su cotidianeidad" me parece una concepción para tensionar. Por cuanto la escuela es parte y se construye con los cotidianos sociales en que transcurre la vida de niños y jóvenes escolarizados y es el lugar del trabajo docente que se realiza con determinados presupuestos, en el contexto de determinadas políticas socioeducativas. Es decir, que se trata de un ámbito que se construye cotidianamente en relación con otros "en procesos dialécticos de mutuas penetraciones y condicionamientos." (Achilli, 2010). La experiencia escolar ingresa a los distintos ámbitos por los que los sujetos escolares (docentes, niños, jóvenes), transitan cotidianamente, entre ellos al grupo familiar, a la vez que se incorporan a la escuela las experiencias vividas en otros espacios: laborales, familiares, en redes amicales, etc. En los denominados "barrios prioritarios" "las prácticas y relaciones experimentadas" (Achilli, 2010) en ese tránsito llevan impresas las huellas de la desigualdad social. Por ello entender al juego, la convivencia, la cultura democrática, como aspectos excluyentes a partir de los cuales se define el espacio escolar, en tanto la conflictividad/los problemas sociales impactan en 
Revista de la Escuela de Ciencias de la Educación, año 10, número 9, enero a diciembre de 2014. PÁginas 149-162. ISSN 1851-6297. ISSN en LINEA 2362-3349. PolíticAS SOCIO-EDUCATIVAS Y TERRITORIO. UNA DESCRIPCIÓN PRELIMINAR, Mariana Beatriz Nemcovsky.

él, supone considerar elementos de una concepción armónica de la escuela, que habría que problematizar y analizar en relación a si contribuye a generar la buscada transformación de la realidad escolar.

A la vez se considera que la escuela debería ser pensada en "cada contexto particular específico. Pensar la escuela en cada territorio, conformando un entramado de instituciones sociales. Pensar la Escuela como institución social implica pensarla construyendo redes para el abordaje de diversos problemas sociales, no porque sea su deber resolverlos, sino porque es su responsabilidad abordarlos. Pensar la Escuela como institución social implica abrir los lazos al entorno para construir una certeza: la escuela no está sola" (17).

Entender la necesidad de pensar la institución escolar en "cada contexto particular, específico", supone una conceptualización orientada a delimitar, y a delimitarse. Se trataría de una escuela anclada en el territorio, ámbito identificado para la construcción "artesanal" de redes entre instituciones, que posibiliten la articulación, a partir de ella, de políticas para que "no esté sola". Ello nos abre algunos interrogantes: si la caracterización del contexto asume tal especificidad que conlleva a "pensar-la escuela en cada territorio", ¿Se está considerando que los problemas sociales aludidos asumen particularidades según el contexto y atraviesan cada espacio escolar con diferencias? Entonces ¿Las articulaciones en el territorio que se puedan generar a partir de la escuela se están entendiendo como distintas y particulares en "cada territorio"? Porque anclar en el territorio supone la confluencia y articulación de las políticas en espacios definidos en función de propósitos determinados. Desde la perspectiva del enfoque territorial de las políticas se sostiene que el interés por esta noción se relaciona "con los aspectos políticos e institucionales que derivan, básicamente de la crisis del Estado y de la pérdida creciente de su poder de regulación... a partir de la ampliación de la capacidad de la economía capitalista de plantear en escala planetaria la interdependencia entre las condiciones de tiempo y espacio en el proceso global de producción de mercancías" (Schneider \& Peyré Tartaruga, 2006). Se entiende la posibilidad de procesos con "una dinámica territorial muy propia"(Coulert \& Pecqueur, 1994 en Schneider et al. 2006) lo que favorece una concepción recortada de los fenómenos locales y el encubrimiento de las "conexiones" históricas de los procesos sociales, en el sentido de Eric Wolf (1993). En esa perspectiva "la descentralización y la valorización de la participación y del protagonismo de los actores de la sociedad civil, especialmente ONG's y los beneficiarios directos de las políticas" (Schneider et al. 2006) resulta consustancial a una lógica fundada en una crisis del estado para regular acciones antes consideradas inherentes a su conformación.

Desde esta concepción entiendo que el trabajo territorial en contextos priorizados, es decir en determinados barrios y zonas de pobreza urbana de la ciudad de Rosario que conlleva un abordaje "artesanal" con su población, y que a la vez supone, como hemos señalado, decisiones en torno a imple- 
Revista de la Escuela de Ciencias de la Educación, año 10, número 9, enero a diciembre de 2014. PÁginas 149-162. ISSN 1851-6297. ISSN EN LINEA 2362-3349. PolitICAS SOCIO-EDUCATIVAS Y TERRITORIO. UNA DESCRIPCIÓN PRELIMINAR. Mariana Beatriz Nemcovsky.

mentaciones "desiguales" entre escuelas, orienta a considerar la continuidad de perspectivas "focalizadas" en las políticas educativas.

En este sentido la concepción de una escuela entendida como parte de un "sistema educativo antiguo y estructuralmente rígido" (18) "que necesita y debe cambiar", que "es una institución de la modernidad resquebrajada" (19), resulta paradojal respecto de la importancia que se le otorga para la acción en el territorio. En tanto herramienta para la construcción del entramado de instituciones para la implementación de políticas y "responsable" de "abordar problemas sociales". Es decir, considerar la "responsabilidad de este abordaje" desde una institución a la vez conceptualizada como debilitada.

\section{Políticas de inclusión y territorio. Procesos en tensión}

El recientemente lanzado Plan Abre (20) [2014] plantea el desarrollo de un conjunto de actividades y planes: el Plan Vuelvo a Estudiar, ya mencionado, el "fomento a clubes de barrio y actividades deportivas, talleres de oficios y capacitación, la jornada ampliada en escuelas primarias, empresas sociales de inclusión, impulso a la economía social en los barrios. Las mismas conforman el eje de convivencia y participación cuyo objetivo es "promover la convivencia, fortalecer las instituciones y acompañar las trayectorias de nuestros jóvenes". Se trata de un conjunto de acciones contempladas "a mayor escala" que desde los gobiernos provincial y municipal se propone implementar "en aquellos sectores de la ciudad donde hay mayor confluencia del delito, la violencia y la inseguridad" (21) y cuya finalidad explícita es "pacificar la ciudad".

Esto es, los jóvenes aparecen fuertemente identificados como destinatarios de las políticas públicas en general y de las políticas socio-educativas en particular. Pero son los jóvenes que habitan en determinados sectores de la ciudad donde confluyen "el delito, la violencia y la inseguridad". La inclusión educativa emerge, entonces, como una estrategia de las políticas dirigidas hacia esa franja de edad para contener "la violencia y la inseguridad" y asegurar la gobernabilidad del territorio. Son los jóvenes de "los sectores más desfavorecidos de la sociedad santafesina" los que aparecen en las formulaciones de las políticas provinciales como su foco explícito. Y son los "barrios prioritarios", patrullados (22) desde abril de 2014 por distintas fuerzas de seguridad nacional, los espacios en los que se superponen políticas públicas que son caracterizadas por los pobladores como de insuficientes, discontinuas en el tiempo y aisladas. Es decir, políticas que involucran acciones en las que, en general, no media un proceso que devenga articulado, y en el tiempo, en el que se planifique, decida y accione de modo conjunto, desde los distintos espacios institucionales correspondientes. Que "pueda ser reconocido por los jóvenes y sus familias como serio" tal como señalan referentes barriales (23).

En este sentido, me parece necesario señalar que durante la segunda mitad de los 90', e inicios de los 2000 la Secretaría de Promoción Social de 
Revista de la Escuela de Ciencias de la Educación, año 10, número 9, enero a diciembre de 2014. Páginas 149-162. ISSN 1851-6297. ISSN en LINEA 2362-3349. PoliticAS SOCIO-EDUCATIVAS Y TERRITORIO. UNA DESCRIPCIÓN PRELIMINAR. Mariana Beatriz Nemcovsky.

la Municipalidad de Rosario, sostenía un conjunto de programas y proyectos socio-educativos (24) consistentes en acciones focalizadas en los barrios de la ciudad, destinados a niños, niñas y jóvenes. Tales programas fueron "cerrados" y/o transformados, de manera tal que, para los grupos sociales que los habitan, ello supone en nuestro presente que "ya no hay más nada" (25).

Ello parece un contrasentido a partir del énfasis puesto en la territorialización, en los diseños de las políticas, que se inicia en esos años y continúa fuertemente. El desarrollo de esos programas, sin embargo, no parece haber favorecido la construcción de las redes interpersonales, interfamiliares, interinstitucionales, que tenían como objetivo explícito. Los procesos de desestructuración de distintos colectivos: familiares, laborales, barriales, entre otros, producida durante la década de transformación neoliberal en nuestro país, fueron coincidentes con la implementación en territorio de dichas acciones focalizadas, en distintas zonas de la ciudad, algunas de pobreza estructural. Ambos procesos entre mutuas conexiones concretas permearon las experiencias de vida de los sujetos favoreciendo la fragilización de antiguas redes de solidaridades y reconstruyendo nuevos lazos de interacción en un clima de época impregnado de aspectos de la lógica hegemónica, como la competitividad y el individualismo. Así las redes de relaciones entre distintos sujetos (trabajo, escuela, familia, barrio) que preexistían a ese fuerte trabajo en territorio, no parecen haber sido re-creadas en la conformación de colectivos que orientaran proyectos con objetivos comunes.

\section{Conclusiones}

La escuela aparece entonces entendida en el cruce de distintos núcleos tensionales:

1) Es conceptualizada como "una institución de la modernidad" "debilitada" "resquebrajada", parte de un sistema "antiguo, rígido", que sin embargo se concibe en las formulaciones de las políticas como el ámbito fundamental a partir del cual se promueve la inclusión educativa como estrategia para la inclusión social.

2) Se la reconoce como espacio distinto/recortado en el contexto barrial, como una institución del estado presente en el territorio a la que se caracteriza como "escenario de la cultura democrática" sobre la que "impactan las problemáticas sociales", "que no está sola" pero a la que se construye armónica y diferencialmente. La conflictividad, que le es inherente en tanto producto de la historicidad, la ubica a la vez como un espacio de relaciones y prácticas con las cuales se lleva adelante una labor, con una continuidad, elementos entendidos como necesarios por los sujetos para incidir favorablemente en la cotidianeidad social. Esos aspectos que, en general, son reconocidos positivamente por los docentes, directivos y los conjuntos sociales y referentes barriales que habitan 
Revista de la Escuela de Ciencias de la Educación, año 10, número 9, enero a diciembre de 2014. PÁginas 149-162. ISSN 1851-6297. ISSN EN LINEA 2362-3349. PolitICAS SOCIO-EDUCATIVAS Y TERRITORIO. UNA DESCRIPCIÓN PRELIMINAR. Mariana Beatriz Nemcovsky.

los "barrios prioritarios", se hacen cotidianamente en un interjuego, en el proceso concreto de múltiples determinaciones.

3) El conjunto de políticas socio-educativas diseñadas y/o implementadas la tienen como eje para su "trabajo artesanal" de construcción de redes, para alcanzar dicha inclusión educativa. No obstante, en su implementación concreta, al producirse de modo parcializado, "seleccionando por escuelas, por aula, en los barrios prioritarios" y según "presupuestos", "priorizando líneas pedagógicas a trabajar", "por niveles" ${ }^{26}$, se generan disrupciones entre escuelas y al interior de ellas. Se favorecen experiencias diferenciales entre los docentes y entre niños/jóvenes y sus grupos familiares. Resultan entonces, aspectos paradojales, contradictorios, en esas implementaciones que se proponen construir articulaciones desde acciones que refuerzan distintas segmentaciones desde políticas focalizadas. Las políticas que asumen esta característica, en los 90', inspiradas en el Consenso de Washington, se enfocaban en determinados grupos sociales y tendían a compensar el corrimiento de las responsabilidades del Estado, con la consigna de reordenar las prioridades del gasto público de áreas como educación y salud pública. En nuestro presente elementos de esas políticas se expresan en la continuidad que supone la construcción de una jerarquización de las necesidades, en las decisiones clasificatorias respecto de los sujetos a los que están destinadas. A la vez, por la fuerte fragmentación que se favorece en el desenvolvimiento concreto de las prácticas, sentidos y relaciones, vinculadas a su implementación /adaptación, suponen lógicas contradictorias con los mismos procesos de articulación e inclusión propuestos.

\section{Notas bibliográficas}

(1) Esta presentación se articula al Proyecto PID Secyt-UNR 2012-2015; Res. C.S. 582/2012 Estado y Transformaciones Urbanas. Un Análisis de Procesos Socioeducativos, Familiares y Laborales; PID-SECYT-UNR (2012-2015); Res. C.S. N 582/2012; Código: HUM 373; Directora: E.Achilli; Co-Dirección.: M. Nemcovsky y J. Shapiro.

(2) Achilli, E (1995) Diagnóstico Socio-demográfico de las seccionales cuarta, dieciséis y once de la ciudad de Rosario. Ceac- UNR.

(3) Según Adriana Rossi, investigadora de la Facultad de Ciencia Política y Relaciones Internacionales de la Universidad Nacional de Rosario en el año 1996 el consumo de cocaína en Rosario superaba la media nacional. Entrevista Televisión Pública (26/02/2014).

(4) "El Gran Rosario, fue por lo tanto, durante toda la década del 90 una región con altas tasas de desocupación, y con una tendencia a la baja en la tasa de empleo. En efecto, el valor más bajo de la serie de los 90 se registra en $1993(10,8 \%)$, la tasa se duplica en mayo de 1995, llegando al 20,9 por ciento, tiene un descenso entre 1997 (16,1 \% en mayo) y 1998 (13,8 \% en mayo). Sin embargo, en mayo del 2000 sigue siendo muy alta $(18,5 \%)$. Por ese motivo, nos concentramos en el análisis de las consecuencias sociales de la desocupación en esta región en la década de los 90 , dado que por la permanencia de estas tendencias, su población ha estado expuesta a una situación de desempleo estructural" En Merlinsky, M.G. (2002) Las consecuencias sociales de la desocupación en Argentina. El desempleo y sus múltiples modos de exclusión. Scripta 
Revista de la Escuela de Ciencias de la Educación, AÑo 10, número 9, enero a diCiembre de 2014. PÁginas 149-162. ISSN 1851-6297. ISSN EN LINEA 2362-3349. PolítICAS SOCIO-EDUCATIVAS Y TERRITORIO. UNA DESCRIPCIÓN PRELIMINAR. Mariana Beatriz Nemcovsky.

Nova . Revista electrónica de Geografía y Ciencias Sociales. Universidad de Barcelona ISSN: 1138-9788. Vol VI, num,119(36), 1 de agosto de 2002.

(5) Entre los programas planteados como de innovación pedagógica se encuentran los denominados: "Rutas Santafesinas"("destinado a jóvenes");"Orquestas y coros infantiles y juveniles" (nivel primario y medio); "Mirada maestra" (docentes en actividad);"Ferias de Ciencia y Tecnología" (todos los niveles y modalidades); "Cátedras experimentales" (Profesorados de Educación inicial y primaria); "Vuelvo a estudiar" (nivel medio); "El patio" (niños, jóvenes y adultos en el barrio); "Escuela Abierta"(Formación docente). Se presentan diversos Programas de Formación continua, entre ellos: Ios de Educación Sexual Integral (nivel inicial, primario y medio); Educación vial; Enseñar la Shoá (nivel medio); "Ronda de palabras"(nivel primario); "Aprendizaje en juego" (nivel inicial). Más información pág Web www. Santafe.gov.ar subportal de educación de la provincia de Santa Fe: http://www.santafe.gov.ar/index.php/educacion/guia/busqueda mayo 2014.

(6) Las modalidades educativas que se desarrollan en el la Provincia de Santa Fe son: la Educación Técnico Profesional, la Educación Artística, la Educación Especial, la Educación Permanente de Jóvenes y Adultos, la Educación Rural y la Educación Intercultural Bilingüe.

(7) En entrevistas (julio-noviembre de 2013)realizadas a docentes y directivas escuela provincial ubicada en el distrito oeste de la ciudad y a la que asisten niños de Villa Banana; San Francisquito; Roullión al 4400; Ituzaingó y Lima; Bajo Hondo.

(8) El proyecto cuenta con un equipo central perteneciente a la Dirección de Políticas SocioEducativas del Ministerio de Educación de la Nación.

(9) Ministerio de Educación de la Provincia de Santa Fe (2014) Programa de Formación docente musical: ensambles, canto y movimiento expresivo. Para más información ver página Web www.santafe.gov.ar Subportal de Educación de la Provincia de Santa Fe.

(10) Ministerio de Educación (2014).Programa Feria de Ciencia. Para más información ver página Web www.santafe.gov.ar Subportal de Educación de la Provincia de Santa Fe.

(11) Ministerio de Educación de la Provincia de Santa Fe (2014) Programa Escuela Abierta Para más información ver página Web www.santafe.gov.ar Subportal de Educación de la Provincia de Santa Fe.

(12) La Dra. Graciela Frigerio (2013) presente en el re-lanzamiento del Programa Sabores y Saberes sostiene que "la propuesta tiene como ejes centrales la "comensalidad, territorialidad y construcción colectiva". Para más información ver página Web www.santafe. gov.ar Subportal de Educación de la Provincia de Santa Fe.

(13) Presentación de Sabores y Saberes a cargo de la Ministra de Educación provincial. Para más información ver página Web www.santafe.gov.ar Subportal de Educación de la Provincia de Santa Fe.

(14) Ministerio de Educación de la Provincia de Santa Fe (2014) Programa El Patio. Para más información ver página Web www.santafe.gov.ar Subportal de Educación de la Provincia de Santa Fe.

(15) Registro entrevistas con docentes y directivas distrito oeste setiembre de 2013.

(16) Ministerio de Educación de la Provincia de Santa Fe (2014) Programa Vuelvo a Estudiar. Para más información ver página Web www.santafe.gov.ar Subportal de Educación de la Provincia de Santa Fe.

(17) "La escuela como institución social: pensar la Escuela como institución social implica no sólo aceptar que la escuela está siendo interpelada por problemáticas sociales que la atraviesan e impactan en su cotidianeidad, sino fundamentalmente pensar la escuela en cada contexto particular específico..." En www.subportal de Educación, Ministerio de Educación de la Provincia de Santa Fe Innovación Pedagógica; Escuela Abierta. 
Revista de la Escuela de Ciencias de la Educación, año 10, número 9, enero a diciembre de 2014. PÁginas 149-162. ISSN 1851-6297. ISSN EN LINEA 2362-3349. PoliticAS SOCIO-EDUCATIVAS Y TERRITORIO. UNA DESCRIPCIÓN PRELIMINAR. Mariana Beatriz Nemcovsky.

(18) Ministerio de Educación de la Provincia de Santa Fe.(2014) Innovación pedagógica www. Santafe.gov.ar subportal de educación de la provincia de Santa Fe.

(19) Entrevista con personal técnico del Ministerio de Educación de la provincia de Santa Fe 22/04/2014.

(20) "Con este Plan vamos a dar mayor escala a las acciones que ya venimos desarrollando en aquellos sectores de la ciudad donde hay mayor confluencia del delito, la violencia y la inseguridad. Son los mismos donde aún faltan infraestructura y servicios y las condiciones de vida son inadecuadas...Asumimos la gran tarea de pacificar a la sociedad rosarina y lo vamos a hacer junto con municipios y organizaciones de la sociedad civil...Sus ejes de trabajo son: Infraestructura y hábitat y Convivencia y participación. Se propone promover la convivencia, fortalecer las instituciones y acompañar las trayectorias de nuestros jóvenes a través de: el Plan Vuelvo a Estudiar; fomento a clubes de barrio y actividades deportivas, Talleres de oficios y capacitación; Jornada ampliada en escuelas primarias; empresas sociales de inclusión, impulso a la economía social en los barrios; Centros territoriales de denuncias; Campañas DNI; Salud integral y prevención de adicciones; mesas de gestión barrial; Ingenia; Colonias de vacaciones". En Plan Abre, Mejores barrios, mejor ciudad, publicación conjunta de la Municipalidad de Rosario, Gobierno de la Provincia de Santa Fe (2014).

(21) "La inversión total en esta fase inicial superará los 76 millones de pesos, destinados básicamente a obras de infraestructura (lo que incluirá apertura de calles, relocalización de hogares, construcción de viviendas, trazado de plazas y provisión de servicios básicos), así como al plan Nueva Oportunidad, un programa de capacitación en oficios y becas que en esta instancia llegará a mil jóvenes de la ciudad: 500 desde la semana próxima y otros tantos a partir de julio" Diario La Capital de Rosario, 30/05/2014.

(22) Desde el 10 de abril de 2014 fuerzas de seguridad federales tales como gendarmería nacional prefectura naval argentina patrullan la ciudad a partir del incremento de homicidios y delitos que en general son vinculados a circuitos de narcocriminalidad.

(23) El padre Daniel Sinieris reconocido referente del Barrio Nuevo Alberdi de la ciudad de Rosario en entrevista canal 5 de Rosario mayo de 2014.

(24) Tal es el caso del Programa CRECER, el Programa Volver al Club de Barrio, Ludotecas, dirigidos a determinados grupos sociales, definidos a partir de determinadas características: edad, género, embarazo, pobreza.

(25) Comentario realizado, durante la espera para la inscripción al Programa Equidad Educativa (Municipalidad de Rosario), por una adolescente que vive en uno de los barrios habitados por el pueblo qom en la ciudad de Rosario, asistente de niña con sus hermanos al Centro Crecer $\mathrm{N}^{\circ} 8$ ubicado en el mismo, a una ex coordinadora de dicho centro (abril de 2014).

(26) A partir de la información que se desprende del sub portal de educación del Ministerio de Educación provincial, de conversaciones con personal de dicho ministerio (22/04/2014) y de entrevistas con docentes y directivas de escuelas del distrito oeste de la ciudad de Rosario (julio-noviembre de 2013).

\section{Referencias Bibliográficas}

- Achilli, E. (2010). Escuela, familia y desigualdad social. Una antropología en tiempos neoliberales. Rosario: Laborde Editor.

- Bernardi, G.; Menna, M. \& Nemcovsky, M. (2013). Sentidos docentes y escolarización infantil en contextos de desigualdad social. En XII Jornadas Rosarinas de Antropología 
Revista de la Escuela de Ciencias de la Educación, año 10, número 9, enero a diciembre de 2014. Páginas 149-162. ISSN 1851-6297. ISSN EN LINEA 2362-3349. POLÍTICAS SOCIO-EDUCATIVAS Y TERRITORIO. UNA DESCRIPCIÓN PRELIMINAR. MARIANA BeAtRIZ NemcovsKY.

Social. ISSN: 1667-9989 Rosario: Departamento de Antropología Socio-cultural Escuela de Antropología UNR.

- Borón, A. (2008). Consolidando la explotación. La Academia y el Banco Mundial contra el pensamiento crítico. Buenos Aires: Espartaco.

- Ferrer, A. (2008). La Economía Argentina. Desde sus orígenes hasta principios del siglo XXI. Buenos Aires: Fondo de Cultura Económica.

- Menéndez, E. (2010). Lo que aparece, lo que no aparece y lo que desaparece: el caso de las violencias. En Achilli, E., Bernardi, G., Nemcovsky, M. \& Shapiro, J. (Eds.), Vivir en la Ciudad. Tendencias estructurales y procesos emergentes, 17- 47. Rosario: Laborde Editor.

- Merlinsky, M. G. (2002). Las consecuencias sociales de la desocupación en Argentina. El desempleo y sus múltiples modos de exclusión. Scripta Nova. Revista electrónica de Geografía y Ciencias Sociales. ISSN: 1138-9788.Vol VI, n¹19 (36), http://www.ub.es/ geocrit/sn/sn119-36.htm Barcelona: Geo Crítica.

- Ministerio de Educación de la Provincia de Santa Fe. Subportal de Educación. www. santafe.gov.ar/index.php/educacion/102759

- Montesinos, M. P. y Sinisi, L. (2009). Entre la exclusión y el rescate. Un estudio antropológico en torno a la implementación de programas socioeducativos. Cuadernos de Antropología Social №29, 43-60, Buenos Aires: FFyL-UBA

- Nemcovsky, M. (2014). Vulnerabilidad, Inclusión y Territorio. Algunas consideraciones preliminares. En Jornadas Internas del Centro de Estudios Antropológicos en Contextos Urbanos. Políticas Públicas en las Últimas Décadas, Rosario: Ceacu (en prensa)

- Schneider, S. \& Peyré Tartaruga, I. (2006). Territorio y Enfoque Territorial: de las referencias cognitivas a los aportes aplicados al análisis de los procesos sociales rurales. En Manzanal, M., Neiman, G. \& Lattuada, M. (Eds). Desarrollo Rural. Organizaciones, Instituciones y Territorio, 71-102, Buenos Aires: Ciccus.

- Wolf, E. (1993). Introducción Europa y la gente sin historia. Buenos Aires: Fondo de Cultura Económica. 1era reimpresión. (original en inglés 1982). 